\title{
Anaesthetic Considerations for Patients with a Vagal Nerve Stimulator
}

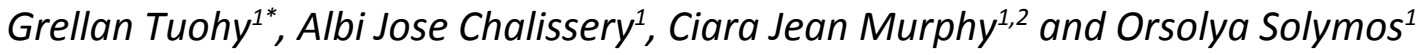 \\ ${ }^{1}$ Connolly Hospital Blanchardstown, Mill Road, Abbotstown, Dublin, Ireland \\ ${ }^{2}$ The Rotunda Hospital, Parnell Square, Dublin, Ireland
}

\begin{abstract}
Vagal nerve stimulation is a neurosurgical treatment modality approved for the management of drug-resistant epilepsy. With its increased utilisation, anaesthesiologists will more frequently encounter patients with an indwelling vagal nerve stimulator (VNS), both in the elective and in the emergency surgical settings. Knowledge of the implications of the indwelling VNS in the perioperative period is vitally important for the anaesthesiologist, both for patient safety and to ensure proper device functioning after any intervention. As there are no published clinical guidelines for the anaesthesiologist on the perioperative management of the patient with an indwelling vagal nerve stimulator, this article will focus on the indications, proposed mechanisms of action and side-effects of vagal nerve stimulation, as well as the anaesthetic considerations for the individual with an implanted vagal nerve stimulator.
\end{abstract}

Keywords

Vagal nerve stimulator, Anaesthesiology

\section{Background}

Vagal nerve stimulation is an important treatment modality which is used as an adjunct in the management for drug-resistant epilepsy (DRE) in Ireland. It is being increasingly utilised in recent years for this indication worldwide, with ongoing research into extending its scope of use for other illnesses and conditions [1].

Approximately $0.5-1.0 \%$ of the population has epilepsy, with the majority of adults presenting with focal onset seizures. Up to $50 \%$ of patients with epilepsy have inadequate control of their seizures or have intolerable side effects of the pharmacological therapy used, such as sedation, impaired concentration and issues in balance and coordination [2]. One in three patients has a poor response to anti-epileptic drugs (AED) from the outset of medical management [3]. There are currently around 40,000 people with epilepsy living in Ireland, and approximately 600 of them have an indwelling VNS.

Drug-resistant epilepsy is defined as the failure of two appropriately chosen and tolerated anti-epileptic drugs (AEDs), whether as monotherapy or in combination. The rate of DRE has not reduced significantly over the last 20 years despite the development of new AEDs with unique mechanisms of action. The consequences of DRE extend beyond seizures, with a potential to cause seizure-related injuries, increased hospital stays, increased mortality and morbidity including sudden unexplained death in epilepsy (SUDEP). DRE also impacts significantly on the individual patient's quality of life resulting in a higher incidence of depression, anxiety, sleep disturbance, cognitive impairment and memory loss. Side-effects with long term AED use are also very common in DRE. Further consequences of DRE include interruption to education or work, and problems with the development and maintenance of interpersonal relationships. The medical and social care of patients with DRE entails significant costs also.

The first VNS was implanted in a patient with refractory epilepsy in 1988 to assess its efficacy [4]. Vagal nerve stimulation was approved as a treatment modality for DRE in Europe in 1994 and in the United States in 1997 [5]. LivaNovaPLC is currently the sole manufacturer of VNS devices worldwide for the treatment of DRE. Chronic, intermittent vagus nerve stimulation has proven to be a safe, effective option for patients suffering from refractory seizures who are not candidates for surgical resection or who hesitate to take the risk of surgery yet continue to have seizures despite maximal medical therapy. Although only a small minority of patients will be entire-

*Corresponding author: Grellan Tuohy, Connolly Hospital Blanchardstown, Mill Road, Abbotstown, D15 X40D, Dublin, Ireland

Accepted: August 08, 2020

Published online: August 10, 2020

Citation: Tuohy G, Chalissery AJ, Murphy CJ, et al. (2020) Anaesthetic Considerations for Patients with a Vagal Nerve Stimulator. J Clin Anesth Pain Manag 4(1):114-122 


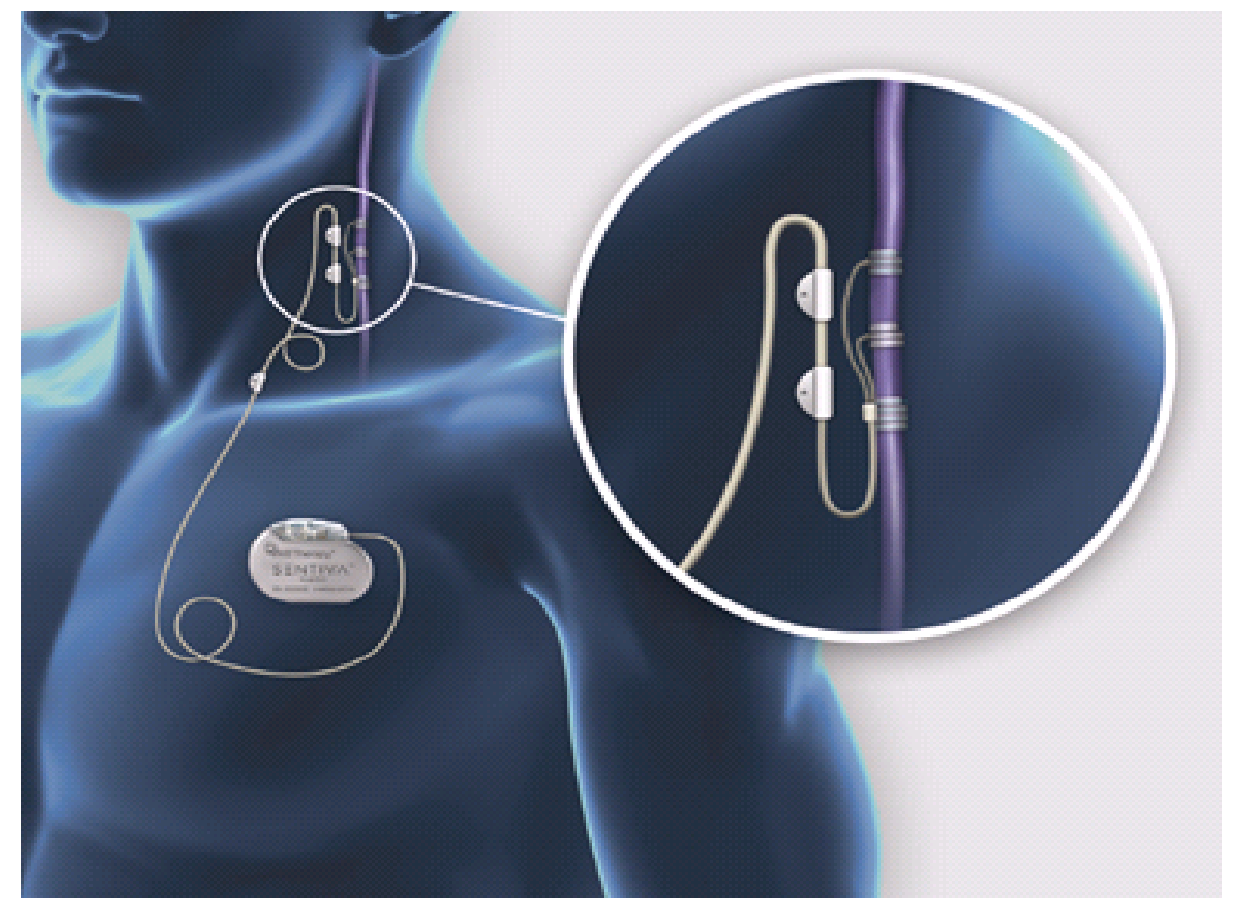

Figure 1: Image of the Sentiva ${ }^{\circledR}$ VNS and its components.

Vagal nerve stimulator components. Digital image: http://us.livanova.cyberonics.com/vnstherapy/how-vns-therapy-works Image credits: LivaNova PLC

ly seizure-free, VNS as an adjunct to medical therapy does appear to significantly improve the quality of life of patients [6]. In two randomized double-blind trials, seizure frequency declined by approximately $30 \%$ after 3 months of treatment. Long-term follow-up studies suggest that response improves over time, with approximately $35 \%$ of patients experiencing a $50 \%$ reduction and $20 \%$ experiencing a $75 \%$ reduction in seizure frequency after 18 months of treatment [7]. A very low number of patients may become medication free or seizure free with VNS. Other benefits reported include improved mood, memory and alertness.

\section{Indications}

Currently in Ireland, DRE is the only approved indication for VNS therapy. However in 2005, VNS therapy was approved by the Food and Drug Administration (FDA) for the treatment of severe, recurrent unipolar and bipolar depression in the United States [8].

Vagal nerve stimulation is under investigation as a potential therapy for other disorders including anxiety and Alzheimer's disease, and has potential applications in obesity and chronic pain syndromes $[5,9]$. Enhancing vagal tone by delivering electrical stimulation to the cervical vagal nerves is emerging as a promising novel therapy in heart failure patients also [10]. Recent studies on neuromodulation revealed the potential of the nervous system to control organ function and re-establish physiological homeostasis during illness, with a growing number of studies showing the beneficial effects of cervical vagal stimulation to control experimental and clinical arthritis, even in patients refractory to current anti-rheumatoid treatments [11].

\section{Vagal Nerve Stimulator Components}

A VNS is a neurocybernetic prosthesis (NCP) system comprising an implantable, multiprogrammable pulse generator that delivers electrical signals to the vagus nerve, as presented in Figure 1. The electrical currents are delivered on a predetermined schedule, or may be initiated by the patient with an external magnet [12]. A VNS is a battery-powered device. The battery life of a VNS generator depends on the settings. On average it will last 3-5 years. Battery replacement or removal of the VNS is a surgical procedure. A schematic drawing of the VNS and its implanted components is shown in Figure 1.

The NCP system consists of:

1. The vagal nerve stimulating electrodes carry the electrical signal from the generator to the vagus nerve. The electrode array consists of two upper helical silicone coils that contain the platinum ribbons for mechanical contact with the nerve fibres, as well as an anchor tether on the inferior end of the electrodes. The helical shape allows the coils to non-traumatically adapt to the shape of the vagus nerve.

The reason that the electrodes are implanted around the left vagus nerve is that the right vagus nerve has more cardiac efferents to the sinoatrial node (involved in the pacemaker function of the heart), whereas the left vagus nerve innervates the atrioventricular node (regulating the force of contraction of the heart muscle as a function of the volume of blood returned to the heart, with less influence over heart rate) $[5,13]$. Randall, et al. showed in dogs that stimulation of the right vagus, compared to the left, caused a greater re- 
duction in heart rate and Woodbury and Woodbury (1990) reported that high-level stimulation of the left vagus had no effect on heart rate $[14,15]$. The use of the right vagus nerve for electrode placement can be considered in scenarios where the left vagus nerve is contraindicated. Some small case series report beneficial reduction in seizure frequency without significant cardiac side effects with right sided implantation [16].

2. The programmable signal generator is similar in appearance to a pacemaker and is implanted in a subcutaneous pocket in the patient's left upper chest, just inferior to the clavicle. The pulse generator produces balanced waveforms at constant current and is powered by a single lithium battery. An antenna inside the generator receives programming signals from the programming wand and then transfers these signals to a microprocessor within the generator, thereby controlling the programmable stimulation variables: Output current (clinically tolerated range: 0-4 $\mathrm{mA}$ ), signal frequency $(1-145 \mathrm{~Hz})$, signal pulse width $(130,250$, $500,750,1,000$ microseconds), signal on time $(7,14$, $21 \mathrm{~s}$ and 30 to 270 s in 30 s steps), signal off time, and magnet-activated stimulus parameters - pulse width, output current, and on time.

3. The programming wand, shown in Figure 2, is used to communicate parameter changes to the pulse generator. It uses radiofrequency signals to non-invasively communicate with the generator. The wand enables adjustment of any of the programmable functions of the generator as clinically indicated [12]. In addition, diagnostic testing informs the physician if there are problems with wand-generator communications, lead impedance, or the programmed current, as well as monitoring battery life. The VNS Therapy ${ }^{\circledR}$ programming software and the VNS Therapy ${ }^{\circledR}$ handheld computer are used to store and retrieve data from the signal generator, with the help of the programming wand.

4. All patients with an indwelling VNS receive a magnet, shown in Figure 3. The magnet can be used for 2 indi-

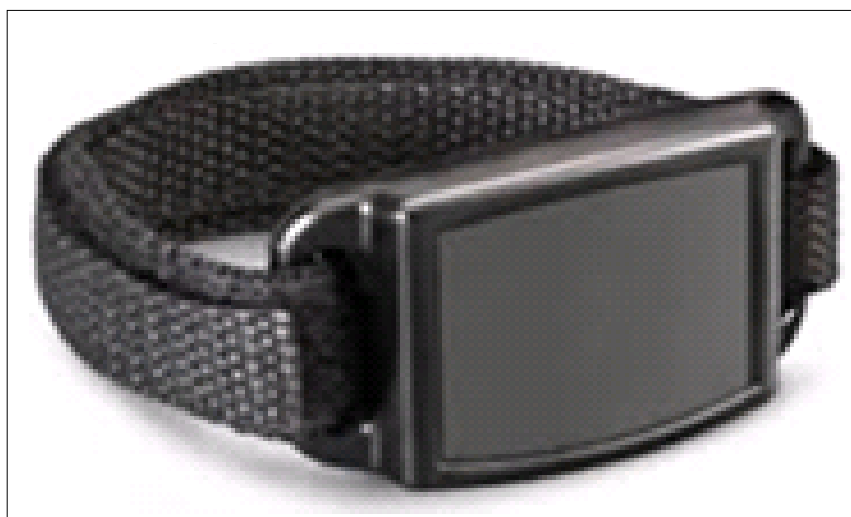

Figure 3: Patient magnet, that all patients with implanted VNS are provided with Magnet bracelet.

Image credits: LivaNova PLC.

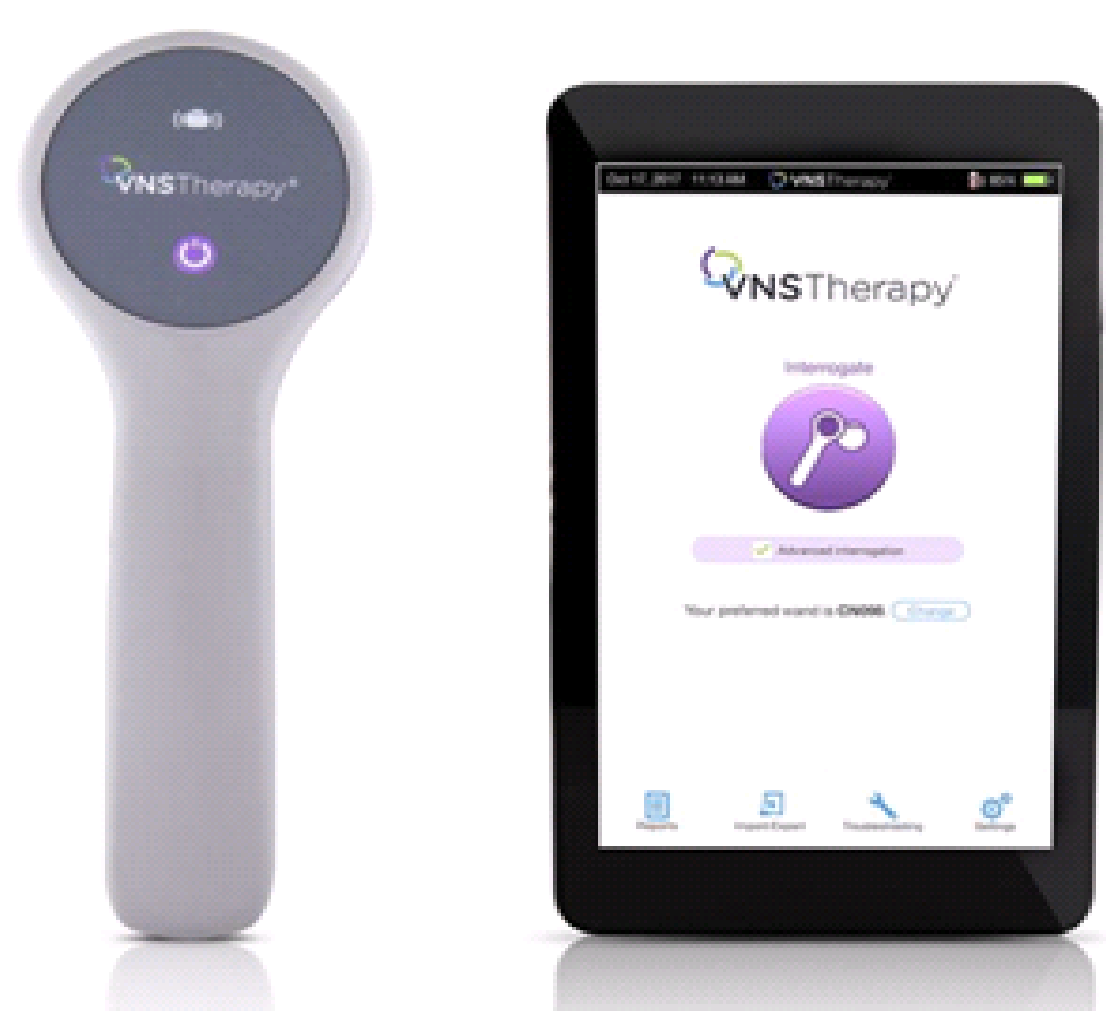

Figure 2: Programming wand with the VNS handheld computer and programming software.

Digital image: http://eplipsy.com/managing-your-epilepsy

Image credits: LivaNova PLC. 
cations, either to deliver an extra vagal nerve stimulation period by brief application of the magnet over the signal generator for $<30$ s or alternatively to stop simulation cycles completely by the continuous application of the magnet for longer than 30 s.

\section{Proposed Mechanism of Action}

The initiation, propagation and maintenance of seizures is a complex pathophysiological process requiring activation and interaction of multiple brain centres to affect duration and spread of the seizure activity. There are a number of proposed mechanisms of action of a VNS and its attenuation of seizure activity. Vagal nerve electrical stimulation creates action potentials within the cervical vagus nerve that modulate cerebral neuronal excitability, either through activation of the limbic system, noradrenergic neurotransmitter systems, or generalized brainstem arousal systems [17]. Altered neurotransmitter expression is evident with an increase in noradrenaline, gamma aminobutyric acid and serotonin levels noted and a decrease in aspartate level [18]. It also increases blood flow in the thalamus and in the cortex. The anticonvulsant effect exerted by vagal nerve stimulation has been described in a variety of animal seizure models. It has no effect on hepatic metabolic processes, serum concentrations of AEDS, or laboratory values. In a study comparing heart rate (HR) variability, it has been shown that VNS does not alter HR variability albeit seizure frequency was lowered. Interestingly, it was shown that HR variability was lower and lacked circadian fluctuations in patients with epilepsy as compared to normal controls. Thus, vagal stimulation does not influence autonomic cardiac control [19].

Takaya, et al. also showed that the anticonvulsant action of vagal nerve stimulation was not solely limited to the stimulation period, because vagal nerve stimulation protected against induced seizures even when discontinued prior to seizure onset, suggesting longer-term changes in neural activity [20]. Furthermore, they investigated the relationship between the cumulative duration of vagal nerve stimulation and its anticonvulsant effect. Awake and freely moving rats were protected by 60 minutes of continuous stimulation from pentylenetetrazol-induced seizures more effectively than 60 minutes of intermittent stimulation with the same current and frequency settings. Similarly, intermittent stimulation was more effective than a single 1-minute stimulus. The anticonvulsant effect was nearly half-maximal 5 minutes after VNS therapy was discontinued.

In summary, current information suggests that VNS therapy activates neuronal networks in the thalamus and other limbic structures and that noradrenaline may mediate the anti-seizure activity of vagal nerve stimulation.

\section{Device Implantation and Programming}

VNS implantation is considered a low-risk surgery with few complications. The implantation procedure usually lasts one to two hours and is typically carried out under general anaesthesia to minimise the possibility that a seizure will affect the operation [21]. VNS implantation can also be performed under the regional anaesthesia technique of combined deep and superficial cervical nerve blocks [22].

Two incisions are made during VNS implantation surgery: A segment of the left vagus nerve is exposed through an approach similar to that used for carotid endarterectomy and a subcutaneous pouch is formed in the upper left chest for the VNS pulse generator [2]. The surgery involves the placement of helical electrodes on the left cervical vagus nerve, with interrogation and testing of the generator to ensure proper function prior to its placement in a subcutaneous pouch. Postoperative neck and chest $\mathrm{x}$-rays may be obtained to confirm placement of the generator and electrode positioning.

The most commonly reported side-effect from the implant procedure is infection. A retrospective review in the United States of 191 paediatric patients with VNS implanted between 2000 and 2007 identified an infection rate of 5.2\% that was consistent with results from prior studies [23]. All infections occurred at the site of the generator placement. The time from implantation to diagnosis ranged from 2 to 93 days (mean 29.8 days, median 18 days). In this review Staphylococcus aureus was the cultured organism in 9 out of the 10 affected patients. This review recommended that all patients be treated as having a deep infection and be given broad-spectrum intravenous antibiotics as first-line treatment.

\section{Programming of VNS}

Ramping up is the term used for increasing the output of the VNS during subsequent appointments after implantation of the device. In Ireland and in the majority of centres in the United States, the VNS is activated two to three weeks after implantation [9]. The treatment plan is to increase the VNS output and currents to a therapeutic level at a speed tolerated by the patient, usually over the course of 8 weeks. Slower ramp up speed may be considered for those considered vulnerable or who are non-verbal. Practice can vary from weekly, fortnightly, monthly ramp up to "rapid" ramp up even, over the course of a week. Ramp up of output current usually takes place in $0.25 \mathrm{~mA}$ increments but this can be increased as tolerated. The therapeutic level of the VNS can vary between patients but will usually be between $1.5 \mathrm{~mA}$ to $2.5 \mathrm{~mA}$, however, this may be lower in some individuals. Of note, after surgically replacing the battery component, the device is switched on immediately postoperatively at a lower than previous stimulation setting.

In the standard mode, the VNS device is set to deliver impulses to the left vagal nerve at regular intervals. Most patients are stimulated at $20-30 \mathrm{~Hz}$, with a stimulation cycle of 30 seconds on, and 5 minutes off [21]. While it is impractical to apply continuous electrical stimulation to the vagal nerve due to safety considerations and limited battery life, the results of Takaya, et al. suggest that the 30 seconds on, 5 minutes off intermittent stimulation protocol utilised for human epilepsy is a reasonable compromise with respect to efficacy, safety, and feasibility [20]. The signals are delivered on a predetermined schedule, or may be initiated by the patient with an external magnet. Once programmed, the generator will deliver intermittent stimulation at the desired settings until any additional programming instructions are received or un- 
til the battery life is expended, which typically occurs after 4 years of operation. In addition, the patient or a companion may activate the generator by placing the supplied magnet over the generator for several seconds; in some patients this may interrupt a seizure or reduce its severity if administered at the onset of the seizure [24].

Some recent developments in vagal nerve stimulation include the auto-stimulation function, whereby a rapid heart rate increase of more than $20 \%$ is detected by the pulse generator and results in the automatic delivery of an unscheduled dose of stimulation. This is further to the observation that 8 in 10 people with epilepsy experience a rapid increase in heart rate before or during a seizure. This closed loop stimulation principle may be of benefit as manual triggering of extra vagal stimulation with the magnet may not always be feasible for a number of reasons such as lack of preceding symptoms, not having the magnet to hand or seizures during sleep. Other events leading to an increase in heart rate, for example the patient exercising, may lead to delivery of an extra dose of vagal nerve stimulation in the 'detect and respond' mode, but this does not harm the patient, as per the manufacturer advice. This auto-stimulation function can however be switched off should the patient wish to receive solely the standard therapy, and it should not be used in patients with clinically significant arrhythmias or in patients who are using treatments that interfere with normal intrinsic heart rate responses, for example patients with pacemaker dependency, patients with implantable defibrillator, patients on beta adrenergic blockers.

\section{Side-Effects and Implications for Anaesthesi- ologists}

Vagal nerve stimulation is safe and well tolerated in patients with longstanding, medically-refractory partial-onset epilepsy. Potential side effects of vagal stimulation could be related to the implantation procedure or during actual stimulation of the vagus nerve [25].

The most frequently encountered adverse effects typically occur during stimulation, are usually mild to moderate in severity, and resolve with reduction in current intensity [2]. These include patients having complaints with tingling or prickling sensation (paraesthesia) and/or vocal symptoms such as hoarseness, sore throat and dry cough which improve over time and with adjustment of stimulation settings [13]. For most patients, side-effects are tolerable and may lessen over time. Serious side-effects are rare.

\section{VNS and cardiac complications}

The intricate relationship of the vagus nerve to cardiac function raises concern that vagal stimulation may affect cardiac rhythm and function. Previous pre-and post-marketing studies have not shown this to be a significant problem, with the incidence of bradyarrhythmias reported to be about $0.1 \%$ [26]. Cardiac complications associated with VNS therapy have been reported mostly in connection with intra-operative lead testing, with the most commonly reported complications being bradycardia and syncope and in rare cases cardiac arrest. 8 cases of bradycardia and asystole have been reported in the literature during lead tests upon device implantation [27]. However, under certain conditions, vagal nerve stimulation can also be proarrhythmic. For example, increased vagal tone could trigger ventricular fibrillation in Brugada syndrome.

Asconape, et al. published a case report in 1999 of a 56-year-old man who developed two episodes of bradycardia and transient asystole during routine lead testing during a planned VNS implantation. While undergoing routine lead diagnostic testing during the implantation procedure, at the initial two stimulations, bradycardia of 30 beats per minute developed. A third stimulation attempt led to the development of transient asystole that required atropine and brief cardiopulmonary resuscitation. The procedure was immediately terminated, with no further sequelae or abnormalities identified on postoperative cardiological evaluation. The possible mechanisms that were contemplated for the bradycardia/asystole included stimulation of cervical cardiac branches of the vagus nerve either by collateral current spread or directly by inadvertent placement of the electrodes on one of these branches; improper plugging of the electrodes into the pulse generator, resulting in erratic varying intensity of stimulation; reverse polarity; and idiosyncratic-type reaction in a hypersusceptible individual [27].

In 2004, Ali, et al. looked at three cases of ventricular asystole with complete heart block that occurred during intraoperative lead tests, to identify the specific type of cardiac abnormality associated with vagus nerve stimulation, and to identify individuals at risk [26]. The authors were able to obtain cardiac rhythm strips during these episodes, which showed a normal, regular $\mathrm{p}$ wave (atrial rhythm) with no ventricular activity, indicating a complete atrioventricular (AV) nodal block. One possible neuroanatomic explanation would be direct activation of the parasympathetic pathway with an exaggerated effect on the AV node. The left vagal nerve has a greater negative chronotropic effect on the atrioventricular node. This effect, however, is normally modified by the sympathetic tone and the stimulation frequency. Clinical activation of the left vagus nerve as with the VNS does not normally have a significant effect on cardiac rhythm; this may explain why this is such a rare occurrence in patients implanted with this device.

Cardiac complications can occur later in the treatment course as evidenced in a case report by Amark, et al. where vagally induced bradyarrhythmia, perfectly correlated with the stimulation periods, suddenly occurred two years and four months after the VNS implantation in a 17-year-old with medically refractory epilepsy [28]. Research is ongoing into the possible long term changes within the central nervous system which may occur. This is based on VNS action via the nucleus tractus solitarius and its projections to other nuclei, further affecting higher autonomic functions in the hypothalamus and insular cortex [26]. This possible mechanism is supported by functional magnetic resonance imaging (fMRI) studies [29].

Even though arrhythmia could be critical, most cases were initially treated as new-onset epileptic seizures with adjustment of the anti-epileptic drugs for a period. To prevent car- 
diac asystole in VNS treatment, clinicians should be alert to the possibility of new-onset syncopal events differing from habitual seizures and investigate them appropriately [30].

\section{VNS and respiratory complications}

Chronic VNS therapy can lead to significant respiratory complications and has been associated with cases of laryngeal dysfunction such as altered voice, dyspnoea and cough. There have also been reports of an increased incidence of obstructive sleep apnoea (OSA) episodes with vagal nerve stimulation.

Previous research has shown that up to one-third of patients with refractory epilepsy has OSA as defined by a respiratory disturbance index (RDI) of $>5$ [31]. Studies of patients with OSA and a VNS showed significantly reduced airflow while sleeping during periods of vagal stimulation with one case associated with recurrent airway obstruction [32]. Vagal nerve stimulation may also cause new onset sleep apnoea episodes in patients who have not previously been diagnosed with this condition. Lower stimulation frequencies, longer periods of off time as well as device inactivation are associated with reduced respiratory events. It is clear that there is a relationship between epilepsy and obstructive sleep apnoea, with patients with VNS therapy having increased apnoeic events in association with stimulation periods. Screening for sleep apnoea events before and after implantation would be prudent as well as careful perioperative risk assessment and management. Perioperative management of patients with OSA and a VNS should aim to minimise post-operative respiratory risks, such as the utilisation of an opiate free anaesthesia technique, close postoperative monitoring in appropriate locations, supplemental oxygen post operatively and discussion with neurology about turning off the VNS for the high risk perioperative period.

Vagal nerve stimulation can lead to abnormal movement of the vocal cords with resultant voice alterations, such as hoarseness, breathlessness and in some cases airway obstruction. Voice alterations are due to concurrent stimulation to two branches of the vagus nerve, the superior and recurrent laryngeal nerves which innervate the muscles of the larynx. This is usually mild and associated only with periods of stimulation, but in some cases it may present with a sore throat or evidence of airway obstruction such as dyspnoea or stridor. Medial deviation of the left vocal cord and arytenoids has been documented on videoendoscopy during vagal nerve stimulation in studies [33]. Similarly, there is a case report of recurrent intraoperative obstructive episodes in a patient in whom a laryngeal mask airway was utilised, with evidence of tetanic contraction of the left sided musculature of the larynx in isolation [34]. This potential complication must be considered when using supra-glottic airways while under general anaesthesia and may require the VNS to be switched off preoperatively to reduce the risk of airway obstruction.

\section{Mechanical and electrical safety}

The VNS can have perioperative implications not just due to its side-effects, but also because of the mechanical and electrical safety aspects of the VNS.
High-frequency stimulation may be associated with tissue damage with long term use [12]. This is a major guiding factor in the selection of stimulation parameters for implantable devices. However, there is no evidence that the stimulation protocols in present clinical use cause pathological changes within the vagus nerve. Furthermore, the helical coils of the stimulating electrodes minimise mechanical damage to the vagus nerve, because less handling of the nerve is required for lead attachment and the helical shape is self-sizing and flexible $[35,36]$.

The NCP system has several additional built-in safety and tolerability features:

- Each stimulation 'ramps up' during the initial $2 \mathrm{~s}$ and 'ramps down' for the final $2 \mathrm{~s}$

- A clamping circuit within the generator prevents more than $14 \mathrm{~V}$ from being delivered to the vagus nerve

- Patients may turn off stimulation by continuously holding the supplied magnet over the generator.

This use of the magnet may become necessary if stimulation becomes intolerable or if the patient anticipates a prolonged period of speaking and does not wish to experience hoarseness or voice change.

The antenna within the generator is controlled by radiofrequency signals. Nonetheless, the generator and the electrode leads are unaffected by microwave transmission, cellular phones, and airport security systems.

Short-wave diathermy, microwave diathermy and therapeutic ultrasound diathermy should not be used in patients with an indwelling VNS. Diagnostic ultrasound is not included in this contraindication.

External defibrillation and electrical cardioversion may damage the generator. If external defibrillation is required, the VNS manufacturer recommends using the lowest amount of appropriate energy during each electrical current delivery and that the defibrillation contact pads be placed as far from the generator and implanted lead as possible. Contact pads should be placed so that current will travel in a vector perpendicular to the VNS system.

\section{Magnetic resonance imaging (MRI)}

The primary risks of MRI are excessive heating of the leads attached to the vagal nerve and damage to the stimulator. Other potential risks posed by MRI in patients with an implanted metallic device include spurious device stimulation due to magnetic field gradients, displacement due to static magnetic field interactions, and disruption of the VNS system [37]. MRI related heating forms the primary risk and is influenced by several factors, such as positioning and configuration of the leads and the pulse generator, field strength of the MRI scan, type of transmit coil, amount of radiofrequency energy, and the anatomic site of scanning [38].

A Dutch study that aimed to evaluate the safety of performing brain MRI scans in patients with an indwelling VNS, looked retrospectively at $97 \mathrm{MRI}$ scans that were performed over a two-year period in 73 patients with an indwelling VNS. 
The indication for most MRI scans was epilepsy related. Patients were scanned according to the manufacturer's guidelines. No patient reported any side-effect, discomfort or pain during or after MRI. In one patient in this study, an 8-year-old boy, a lead break was detected based on device diagnostics after the MRI scan. However, with the lack of device diagnostics prior to the MRI scan, it was impossible to rule out if the lead break was already present prior to the MRI scan, or alternatively, if MRI scanning might have been responsible for the lead break due to exposure to the radiofrequency fields. In this published Dutch case series, 11 patients younger than 12 years of age with indwelling VNS had MRI scans without complications. Moreover, this study found that the acquisition of multiple MRI studies in a single patient, even up to nine scans in 3 years, was deemed to be safe. Of note, the study mentions that one patient whose pulse generator had been removed previously, was excluded from having his MRI scan, because of a potentially higher risk of lead heating [37].

Detailed guidelines around the management of VNS for MRI are available from 'MRI Guidelines for VNS Therapy', 2017 edition, created by LivaNova PLC. As a general rule, the generator should be switched off before entering the MRI, and reprogrammed after scanning, to ensure continued appropriate parameters and proper functioning. Depending on the VNS model, exclusion zones for MRI must be observed from C7-L3 vertebrae.

Due to the complexity of patients with DRE and the fact they often have other significant comorbidities, it is a patient group often referred to pre-operative assessment clinic for review by an anaesthesiologist. Table 1 presents a summary of the anaesthetic implications of a VNS and suggested basic managements protocols to be used as a guide. Expert advice should be sought from neurology colleagues for specific advice around peri-operative management and patients should have the pulse generator interrogated after any unplanned or emergency deactivation with the magnet to ensure proper function and appropriate programming going forward.

\section{Conclusion}

Vagal nerve stimulator therapy has been utilised for the management of drug resistant epilepsy since 1988 and it has an expanding scope of use outside of refractory epilepsy. In the hospital where the authors work, patients with an indwelling VNS are not an infrequent occurrence on the dental procedure list for adults requiring general anaesthesia. With the incidence of VNS implantation on the rise, more and more anaesthesiologists will encounter patients with an indwelling VNS, both in the elective and in the emergency setting. Familiarity with the device is important, both for patient safety as well as for avoidance of damage to the device. Expert advice should be sought in the perioperative period to further ensure these goals.

In an effort to raise more awareness of the VNS device and its implications for the anaesthesiologist, we share an overview of the components, physiology and potential side effects associated with its use, focusing on the perioperative period

Table 1: Implications of VNS for anaesthesiologists.

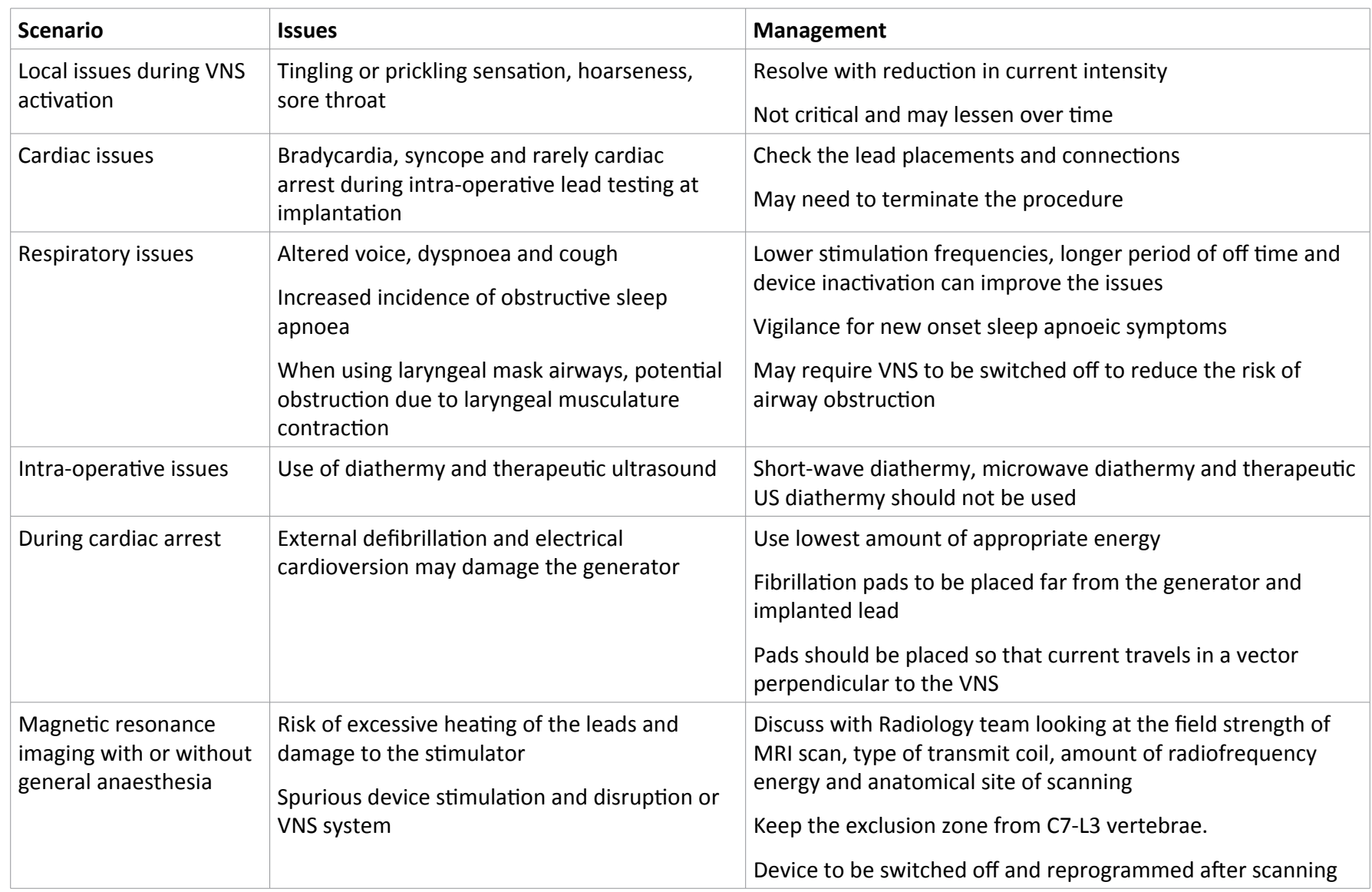


and its specific considerations.

\section{Acknowledgements}

The authors would like to extend their gratitude to Patricia Ennis and Breege Staunton, Epilepsy Surgery Specialist Nurses in Beaumont Hospital Dublin, for providing information on VNS therapy, and to Darren McCarthy, the Territory Manager of the Neuromodulation Division of LivaNova PLC, the supplier of VNS devices in Ireland, for his contribution in providing us with verbal and written information on VNS.

\section{Conflict of Interest}

The authors declare no conflict of interest.

\section{References}

1. Ogbonnaya S, Kaliaperumal C (2013) Vagal nerve stimulator: Evolving trends. J Nat Sci Biol Med 4: 8-13.

2. Schachter SC (2005) Vagus nerve stimulation: Current status and clinical applications. Expert Opin Investig Drugs 6: 1327-1335.

3. Brodie MJ (2013) Road to refractory epilepsy: The Glasgow story. Epilepsia 54: 5-8.

4. Penry JK, Dean JC (1990) Prevention of intractable partial seizures by intermittent vagal stimulation in humans: Preliminary results. Epilepsia 31: S40-S43.

5. Groves DA, Brown VJ (2005) Vagal nerve stimulation: A review of its applications and potential mechanisms that mediate its clinical effects. Neurosci Biobehav Rev 29: 493-500.

6. Milby $\mathrm{AH}$, Halpern $\mathrm{CH}$, Baltuch $\mathrm{GH}$ (2008) Vagus nerve stimulation for epilepsy and depression. Neurotherapeutics 5: 75-85.

7. Cohen Gadol AA, Britton JW, Wetjen NM, et al. (2003) Neurostimulation therapy for epilepsy: Current modalities and future directions. Mayo Clinic Proceedings 78: 238-248.

8. O'Reardon JP, Cristancho P, Peshek AD (2006) Vagus nerve stimulation (VNS) and treatment of depression: To the brainstem and beyond. Psychiatry (Edgmont) 3: 54-63.

9. Fahy BG (2010) Intraoperative and perioperative complications with a vagus nerve stimulation device. J Clin Anesth 22: 213-222.

10. Zhang Y, Mazgalev TN (2011) Arrhythmias and vagus nerve stimulation. Heart Failure Reviews 16: 147-161.

11. Kanashiro A, Bassi GS, Queiróz Cunha F de, et al. (2018) From neuroimunomodulation to bioelectronic treatment of rheumatoid arthritis. Bioelectron Med (Lond) 1: 151-165.

12. Terry RS, Tarver WB, Zabara J (1991) The implantable neurocybernetic prosthesis system. Pacing Clin Electrophysiol 14: 86-93.

13. Hatton KW, McLarney JT, Pittman T, et al. (2006) Vagal nerve stimulation: Overview and implications for anesthesiologists. Anesth Analg 103: 1241-1249.

14. Randall WC, Ardell JL (1985) Selective parasympathectomy of automatic and conductile tissues of the canine heart. Am J Physiol 248: 61-68.

15. Woodbury DM, Woodbury JW (1990) Effects of vagal stimulation on experimentally induced seizures in rats. Epilepsia 31: S7-S19.

16. McGregor A, Wheless J, Baumgartner J, et al. (2005) Right-sided vagus nerve stimulation as a treatment for refractory epilepsy in humans. Epilepsia 46: 91-96.
17. McLachlan RS (1997) Vagus nerve stimulation for intractable epilepsy: A review. J Clin Neurophysiol 14: 358-368.

18. Ben-Menachem E, Hamberger A, Hedner T, et al. (1995) Effects of vagus nerve stimulation on amino acids and other metabolites in the CSF of patients with partial seizures. Epilepsy Res 20: 221-227.

19. Ronkainen E, Ansakorpi H, Huikuri HV, et al. (2005) Suppressed circadian heart rate dynamics in temporal lobe epilepsy. Journal of Neurology, Neurosurgery, and Psychiatry 76: 1382-1386.

20. Takaya M, Terry WJ, Naritoku DK (1996) Vagus nerve stimulation induces a sustained anticonvulsant effect. Epilepsia 37: 11111116.

21. Krahl S (2012) Vagus nerve stimulation for epilepsy: A review of the peripheral mechanisms. Surg Neurol Int 3: S47-S52.

22. Bernard EJ, Passannante AN, Mann B, et al. (2002) Insertion of vagal nerve stimulator using local and regional anesthesia. Surg Neurol 57: 94-98.

23. Air EL, Ghomri YM, Tyagi R, et al. (2009) Management of vagal nerve stimulator infections: Do they need to be removed? J Neurosurg Pediatr 3: 73-78.

24. Hammond EJ, Uthman BM, Reid SA, et al. (1992) Electrophysiological studies of cervical vagus nerve stimulation in humans: I. EEG effects. Epilepsia 33: 1013-1020.

25. Sackeim HA, Rush AJ, George MS, et al. (2001) Vagus nerve stimulation (VNSTM) for treatment-resistant depression: Efficacy, side effects, and predictors of outcome. Neuropsychopharmacology 25: 713-728.

26. Ali II, Pirzada NA, Kanjwal Y, et al. (2004) Complete heart block with ventricular asystole during left vagus nerve stimulation for epilepsy. Epilepsy Behav 5: 768-771.

27. Asconapé JJ, Moore DD, Zipes DP, et al. (1994) Bradycardia and asystole with the use of vagus nerve stimulation for the treatment of epilepsy: A rare complication of intraoperative device testing. Epilepsia 40: 1452-1454.

28. Åmark P, Stödberg T, Wallstedt L (2007) Late onset bradyarrhythmia during vagus nerve stimulation. Epilepsia 48: 1023-1025.

29. Narayanan JT, Watts R, Haddad N, et al. (2002) Cerebral activation during vagus nerve stimulation: A functional MR study. Epilepsia 43: 1509-1514.

30. Kato H, Fujimoto A, Okanishi T, et al. (2018) New onset syncopal events following vagus nerve stimulator implantation might be key to preventing vagus nerve stimulation-induced symptomatic bradycardia - A case report and review. Epilepsy Behav Case Rep 10: $57-60$.

31. Malow BA, Levy K, Maturen K, et al. (2000) Obstructive sleep apnea is common in medically refractory epilepsy patients. Neurology 55: 1002-1007.

32. Malow BA, Edwards J, Marzec M, et al. (2001) Vagus nerve stimulation reduces daytime sleepiness in epilepsy patients. Neurology 57: 879-884.

33. Zalvan C, Sulica L, Wolf S, et al. (2003) Laryngopharyngeal dysfunction from the implant vagal nerve stimulator. Laryngoscope 113: 221-225.

34. Bernards CM (2004) An unusual cause of airway obstruction during general anesthesia with a laryngeal mask airway. Anesthesiology 100: 1017-1018. 
35. Tarver WB, George RE, Maschino SE, et al. (1992) Clinical experience with a helical bipolar stimulating lead. Pacing Clin Electrophysiol 15: 1545-1556.

36. Agnew WF, McCreery DB (1990) Considerations for safety with chronically implanted nerve electrodes. Epilepsia 31: S27-S32.

37. De Jonge JC, Melis GI, Gebbink TA, et al. (2014) Safety of a dedi- cated brain MRI protocol in patients with a vagus nerve stimulator. Epilepsia 55: e112-e115.

38. Shellock FG, Begnaud J, Inman DM (2006) Vagus nerve stimulation therapy system: In vitro evaluation of magnetic resonance imaging-related heating and function at 1.5 and 3 tesla. Neuromodulation 9: 204-213.

DOI: $10.36959 / 377 / 340$

Copyright: (C) 2020 Tuohy G, et al. This is an open-access article distributed under the terms of the Creative Commons Attribution License, which permits unrestricted use, distribution, and reproduction in any medium, provided the original author and source are credited. 